\title{
Mixed convection Flow Along a Horizontal Permeable Lpate in Porous Medium With Variable Permeability-Surface Temperature And Concentration
}

\author{
Amir S. Dawood and Saddam A. Mohammad \\ Department of Mechanical Engineering \\ University of Mosul, Iraq
}

\begin{abstract}
The non-Darcy mixed convection from a horizontal permeable surface embedded in a saturated porous medium with the simultaneous heat and mass transfer has been studied. Uniform and variable permeability effects are also investigated. Variable surface temperature and concentration was considered as a surface condition. Nonsimilar governing equations are obtained by using a suitable transformation and solved numerically by a finite difference method. It is observed that for uniform permeability surface fluid suction and increasing the power law index, thermal dispersion parameter, and buoyancy ratio increases the heat and mass transfer rates. Surface fluid injection and increasing the inertia effect parameter have opposite effect. Increasing Lewis number decreases the heat transfer rate and increases the mass transfer rate. For any particular parameter, variable permeability enhances the heat and mass transfer rates.
\end{abstract}

Keywords: Porous medium, Mixed convection, Horizontal plate, Nonsimilarity solutions, Heat and mass transfer.

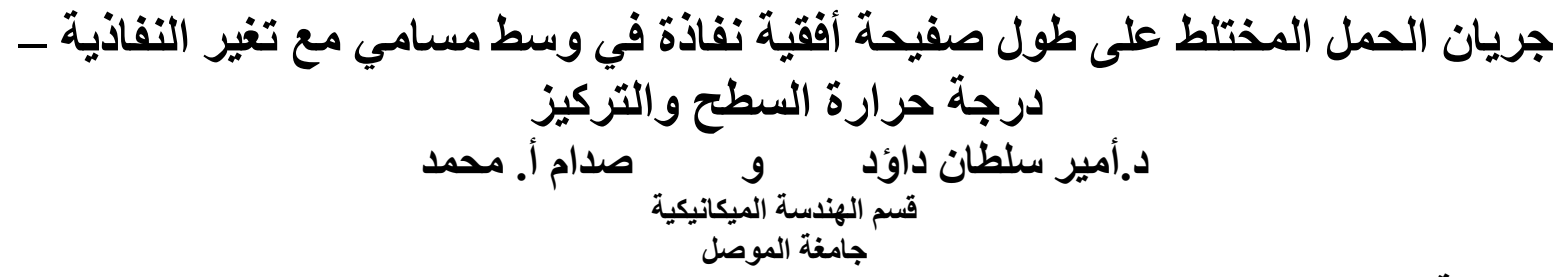

الخلاصة

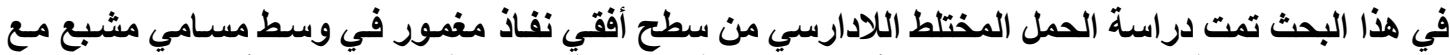

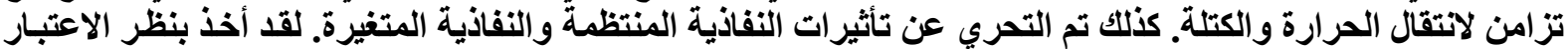

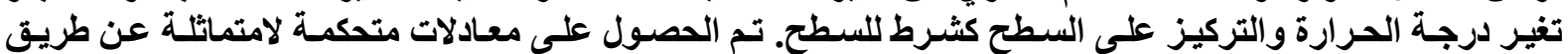

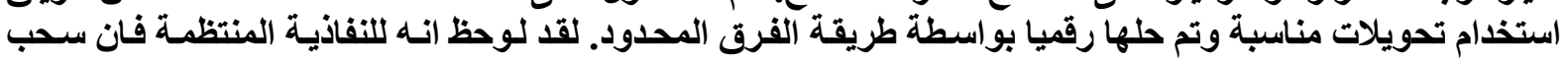

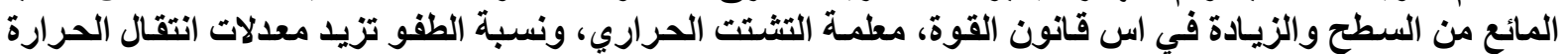

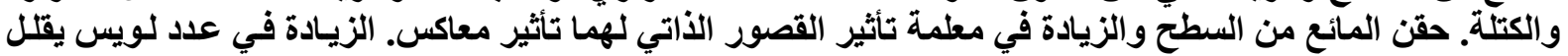

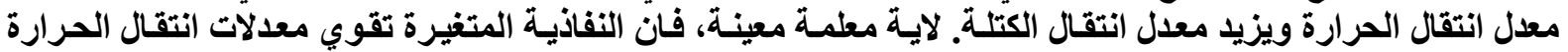
و والكتلة. 


\section{Nomenclature}

\begin{tabular}{|c|c|c|c|}
\hline$a_{1} \& a_{2}$ & Constants, equation (7). & \multicolumn{2}{|c|}{ Greek letters } \\
\hline$A_{1} \rightarrow A_{6}$ & Coefficients, equation (16). & $\alpha$ & Thermal diffusivity of fluid $\left(m^{2} / s\right)$. \\
\hline$b$ & $\begin{array}{l}\text { Inertia coefficient (Ergun's correlation) defined } \\
\text { in equation (3) }(1 / m) \text {. }\end{array}$ & $\alpha_{e}$ & $\begin{array}{l}\text { Effective thermal diffusivity of } \\
\text { the porous medium }\left(\mathrm{m}^{2} / \mathrm{s}\right)^{\text {. }}\end{array}$ \\
\hline$B_{1} \rightarrow B_{12}$ & Coefficients, equation (17). & $\beta_{C}$ & Coefficient of concentration expansion $\left(\mathrm{m}^{3} / \mathrm{kg}\right)$. \\
\hline$c$ & Specific heat of fluid $(J / \mathrm{kg} \cdot K)$. & $\beta_{T}$ & Coeff. of thermal expansion $(1 / K)$. \\
\hline$C$ & Concentration $\left(\mathrm{kg} / \mathrm{m}^{3}\right)$ & $\gamma$ & Parameter defined in equation (12). \\
\hline$d \& d^{*}$ & Constants, equation (9). & $\delta_{T}$ & Thermal boundary layer thickness $(m)$. \\
\hline$d_{p}$ & Particle diameter $(m)$. & $\Delta T$ & $\begin{array}{l}\text { Temperature difference } \\
{\left[=T_{w}(x)-T_{\infty}\right](K)}\end{array}$ \\
\hline$D$ & $\begin{array}{l}\text { Mass diffusivity of the porous } \\
\text { medium }\left(\mathrm{m}^{2} / \mathrm{s}\right) \text {. }\end{array}$ & $\Delta \zeta$ & Subinterval in the $\zeta$-direction. \\
\hline$D_{s}$ & $\begin{array}{l}\text { Thermal dispersion parameter } \\
\left.=\left(d_{n} / x\right)\left(P e_{x}^{1 / 2}+R a_{x}^{1 / 3}\right)^{2}\right]\end{array}$ & $\Delta \eta$ & Subinterval in the $\eta$-direction. \\
\hline$f$ & Dimensionless stream function. & $\zeta$ & Nonsimilarity parameter. \\
\hline$f_{w}$ & $\begin{array}{l}\text { Surface mass flux parameter } \\
\left\{=\left(-V_{w} x\right) /\left[\alpha\left(P e_{x}^{1 / 2}+R a_{x}^{1 / 3}\right)\right]\right\}\end{array}$ & $\eta$ & Pseudosimilarity variable. \\
\hline$g$ & Gravitational acceleration $\left(m / s^{2}\right)$ & $\theta$ & Dimensionless temperature. \\
\hline$h$ & $\begin{array}{l}\text { Local heat transfer coefficient } \\
\left\{=q_{w} /\left[T_{w}(x)-T_{\infty}\right]\right\}\left(W / m^{2} \cdot K\right) .\end{array}$ & $\kappa$ & Coefficient, equation (9). \\
\hline$h_{m}$ & $\begin{array}{l}\text { Local mass transfer coefficient } \\
\left\{=m_{w} /\left[C_{w}(x)-C_{\infty}\right]\right\}(m / s)\end{array}$ & $\lambda$ & $\begin{array}{l}\text { Thermal conductivity ratio of the fluidphase to that } \\
\text { of the solid phase. }\end{array}$ \\
\hline$i$ & Index of mesh points in the $\zeta$-direction. & $\mu$ & Dynamic viscosity of fluid $(\mathrm{kg} / \mathrm{s} \cdot \mathrm{m})$ \\
\hline$j$ & Index of mesh points in the $\eta$-direction. & $v$ & Kinematic viscosity of fluid $\left(m^{2} / s\right)$ \\
\hline$k$ & Thermal conductivity of fluid $(W / m \cdot K)$. & $\rho$ & Density of fluid $\left(\mathrm{kg} / \mathrm{m}^{3}\right)$ \\
\hline$K$ & Permeability of porous medium $\left(\mathrm{m}^{2}\right)$ & $\sum$ & Prefix indicating summation. \\
\hline Le & Lewis number $(=\alpha / D)$ & $\tau_{w}$ & Local wall shear stress $\left(\mathrm{kg} / \mathrm{m} \cdot \mathrm{s}^{2}\right)$. \\
\hline$m_{w}$ & Local surface mass flux $\left(\mathrm{kg} / \mathrm{m}^{2} \cdot \mathrm{s}\right)$ & $U$ & Velocity component $y$-direction $(\mathrm{m} / \mathrm{s})$. \\
\hline$n$ & Constant, equation (7). & $\phi$ & Porosity of the porous medium. \\
\hline$N$ & $\left\{=\beta_{C}\left[C_{w}(x)-C_{\infty}\right] / \beta_{T}\left[T_{w}(x)-T_{\infty}\right]\right\}$ & $\Phi$ & Dimensionless concentration. \\
\hline$N u_{r}$ & Local Nusselt number $(=h x / k)$. & $\psi$ & Stream function. \\
\hline$N_{\zeta} \& N_{\eta}$ & Integer numbers greater than zero. & \multicolumn{2}{|c|}{ Subscripts } \\
\hline$P e_{r}$ & Local Peclet number $\left(=U_{\infty} x / \alpha\right)$. & $e$ & Effective \\
\hline$q_{w}$ & Local surface heat flux $\left(W / m^{2}\right)$ & $n a$ & $\begin{array}{l}\text { Assumed value not affected by the } \\
\text { firstconvergence criterion. }\end{array}$ \\
\hline$R$ & $\begin{array}{l}\text { [nertia effect parameter } \\
\left.=\left(K_{\infty} b_{\infty} \alpha / v x\right)\left(P e_{r}^{1 / 2}+R a_{r}^{1 / 3}\right)^{2}\right]\end{array}$ & new & New value. \\
\hline$R a_{x}$ & {$\left[=K_{\infty} g \beta_{T} x \Delta T \rho_{\infty} /(\alpha \mu)\right]$} & old & Old value. \\
\hline$S h_{x}$ & Local Sherwood number $\left(=h_{m} x / D\right)$. & $s$ & Dispersive. \\
\hline$T$ & Temperature $(K)$ & SO & Solid phase. \\
\hline$u$ & $\begin{array}{l}\text { Velocity component in the } x \text { direction } \\
(\mathrm{m} / \mathrm{s}) \text {. }\end{array}$ & $s t$ & Stagnant. \\
\hline$U_{\infty}$ & Free stream velocity $(m / s)$ & $w$ & Surface conditions. \\
\hline$V_{w}$ & $\begin{array}{l}\text { Surface blowing or suction velocity } \\
(\mathrm{m} / \mathrm{s}) \text {. }\end{array}$ & $\infty$ & Free stream conditions. \\
\hline$x$ & Axial coordinate $(m)$ & & \\
\hline$y$ & Normal coordinate $(m)$. & & \\
\hline
\end{tabular}




\section{Introduction}

In recent years, much work has been dedicated to the area of convective heat transfer in porous media because of its relevance to a variety of situations occurring in engineering and nature. Among these works, natural and forced convection studies occupy the majority of investigations. The interfacial area of mixed convection which connect natural and forced convection, in comparison, has not been given due attention in porous media [1]. The convection heat transfer phenomena in nature are often accompanied by mass transfer, that is, by the transport of a certain substance that acts as a component (constituent, species) in the fluid mixture. Convection mass transfer processes alone (in the absence of heat transfer) constitute the backbone of many operations in the chemical industry. This seems like enough reason to include mass transfer in the studies. An additional argument in favor of this decision is the analogy that exists between convective mass transfer and convective heat transfer [2].

Simultaneous heat and mass transfer from different geometries embedded in porous media has many engineering and geophysical applications. These applications include migration of water in geothermal reservoirs, underground spreading of chemical wastes and other pollutants, thermal insulation, enhanced oil recovery, packed-bed catalytic reactors, cooling of nuclear reactors, grain storage, and evaporative cooling and solidification [3].

When the flow velocity and the pores of the porous medium matrix are small, the porous medium can be modeled by the Darcy law, which assumes a linear empirical relation between the Darcian velocity and the pressure drop across the porous medium. To acquire a better understanding of convective heat transfer in a porous medium, incorporating the effects of various non-Darcy flow phenomena, which are often encountered in many practical situations, into the analysis is necessary. These non-Darcy effects include boundary viscous resistance, flow inertia force, near-wall porosity variation, and thermal dispersion (A secondary effect of a porous medium on the flow appears as a result of mixing and recirculation of local fluid particles through tortuous paths formed by the porous medium solid particles, this effect is classified as thermal dispersion [3]). Inclusion of these nonDarcy effects in the analysis is essential for porous media with a higher flow velocity and/or larger pore sizes, where Darcy's law is inadequate [4].

Previous works on mixed convection over horizontal surfaces(4-14) were done without taking into consideration the convection mass transfer process in the analysis (concentration equation and its relevant boundary conditions). Previous works were carried out for impermeable surfaces (i.e., there was no surface blowing or suction velocity). Furthermore, variation of permeability and porosity of the porous medium was included in the previous studies without including the non-Darcian effects in the analysis with these variations.

In this work the problem that will be considered is the simultaneous heat and mass transfer by mixed convection from a semi-infinite horizontal permeable surface embedded in a fluid-saturated porous medium and in the presence of :

1. Surface blowing or suction.

2. The porous medium inertia and thermal dispersion effects.

3. Incorporating the variation of permeability and thermal conductivity due to packing of particles. concentration.

This will be done for power law variations of the surface temperature and 


\section{Problem formulation}

Consider steady, laminar coupled heat and mass transfer by mixed convection flow of fluid over a semi-infinite permeable horizontal plate embedded in a fluid-saturated porous medium. The coordinate system is shown in Figure (1). For the mathematical analysis of the problem, we assume that gravitational acceleration $(g)$ is acting downward in the negative $y$-direction. Fluid is assumed to be incompressible, Newtonian, and has constant properties except the density in the buoyancy term of the balance of the momentum equation that is approximated according to the Boussinesq approximation. The surface temperature and concentration are assumed to have power law variations with the horizontal distance along the plate. The temperature and concentration of the free stream are assumed to be constant. The temperature and concentration at the plate surface are always greater than the free stream values existing far from the plate surface. Permeability and thermal resistance are functions of the vertical coordinate $y$. The flow is assumed to be two-dimensional and the porous medium is assumed to be non-deformable. At any point in the porous medium, the solid matrix is in thermal equilibrium with the fluid filling the pores. Under these assumptions and the application of Boussinesq and boundary layer approximations, the governing equations are given by [22]:

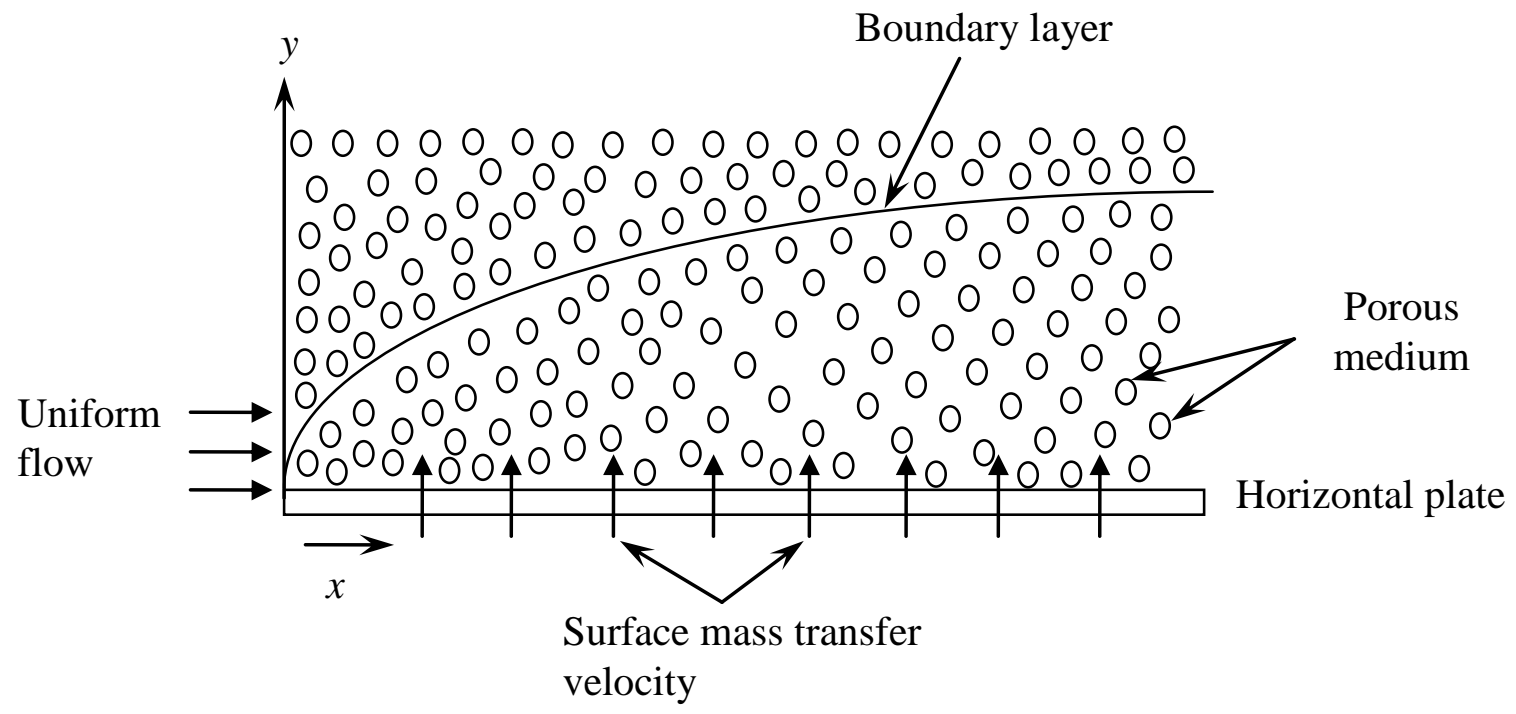

Figure (1): Mixed convection flow adjacent to a permeable horizontal plate embedded in a saturated porous medium.

1. Continuity equation.

$\frac{\partial u}{\partial x}+\frac{\partial v}{\partial y}=0$

Where $u$ and $v$ are the velocity components in the $x$ and $y$ directions respectively.

2. Momentum equation.

$\left[1+\frac{2 b \rho_{\infty} K u}{\mu}\right] \frac{\partial u}{\partial y}=\frac{u}{K} \frac{\partial K}{\partial y}-\frac{\rho_{\infty} u^{2} K}{\mu} \frac{\partial b}{\partial y}-\frac{g \rho_{\infty} K}{\mu}\left[\beta_{T} \frac{\partial T}{\partial x}+\beta_{C} \frac{\partial C}{\partial x}\right]$ 
Where $\rho_{\infty}, \mu, \beta_{T}$, and $\beta_{C}$ are the density, dynamic viscosity, thermal expansion coefficient, and concentration expansion coefficient of the fluid respectively; $K$ is the permeability of the porous medium; $T, C$, and $g$ are the temperature, concentration, and gravitational acceleration respectively; $b$ represents an Ergun 's correlation for a column of packed spheres, it is given as [2]:

$$
b=\frac{1.75(1-\phi)}{\left(\phi^{3} d_{p}\right)}
$$

Where $\phi$ and $d_{p}$ refer to porosity of the porous medium and particle diameter respectively.

3.Energy equation.

$u \frac{\partial T}{\partial x}+v \frac{\partial T}{\partial y}=\alpha_{e} \frac{\partial^{2} T}{\partial y^{2}}+\frac{\partial T}{\partial y} \frac{\partial \alpha_{e}}{\partial y}$

4.Concentration equation.

$u \frac{\partial C}{\partial x}+v \frac{\partial C}{\partial y}=D \frac{\partial^{2} C}{\partial y^{2}}$

Where $\alpha_{e}$ and $D$ are the effective thermal diffusivity and the mass diffusivity of the porous medium respectively.

5. Boussinesq approximation.

As stated earlier the properties of the fluid are assumed to be constant except the density variation in the buoyancy force, which is approximated according to the Boussinesq approximation. This variation, due to both temperature and concentration gradients, can be described by the following equation [15]:

$\rho=\rho_{\infty}\left[1-\beta_{T}\left(T-T_{\infty}\right)-\beta_{C}\left(C-C_{\infty}\right)\right]$

6. Boundary conditions.

The boundary conditions for the problem of power law variations of surface temperature and concentration can be written as:

$$
\begin{aligned}
& y=0: \quad v=V_{w} \quad T=T_{w}(x)=T_{\infty}+a_{1} x^{n} \quad C=C_{w}(x)=C_{\infty}+a_{2} x^{n} \\
& y \rightarrow \infty: \quad u=U_{\infty} \quad T=T_{\infty} \quad C=C_{\infty}
\end{aligned}
$$

In equations ( $7 \& 8), T_{w}$ and $C_{w}$ are the surface temperature and concentration, respectively. $V_{w}$ is the surface blowing or suction velocity; $U_{\infty}, T_{\infty}$, and $C_{\infty}$ are the free stream velocity, temperature, and concentration, respectively; $a_{1}, a_{2}$, and $n$ are constants. 
7. Permeability and porosity.

It will be assumed that the permeability and porosity of the porous medium vary exponentially from the surface due to packing of particles next to the surface $[14,16-19]$.

$$
K=K_{\infty}\left(1+d e^{-y / \kappa}\right) \quad \phi=\phi_{\infty}\left(1+d^{*} e^{-y / \kappa}\right)
$$

Where $K_{\infty}$ and $\phi_{\infty}$ are the permeability and porosity at the edge of the boundary layer, respectively. $d$ and $d^{*}$ are constants, whose values are taken 3.0 and 1.5 [14,16-19], respectively. $\kappa$ will be explained with the proceed in the analysis. The porosity of a packedsphere bed decreases from a value of $0.9 \sim 1.0$ at the surface to $0.38 \sim 0.4$ in the bulk of the bed [4].

8. Effective thermal diffusivity.

The quantity $\alpha_{e}=k_{e} /\left(\rho_{\infty} c\right)$ in equation (4) is the effective thermal diffusivity of the porous medium, with $k_{e}$ denoting the effective thermal conductivity of the saturated porous medium and $\left(\rho_{\infty} c\right)$ the product of density and specific heat of the fluid. The effective thermal conductivity $k_{e}$ can be written as $[4,8]$ :

$$
k_{e}=k_{s t}+k_{s}
$$

Where $k_{s t}$ is the stagnant thermal conductivity and $k_{s}$ is the thermal dispersive conductivity. The stagnant thermal conductivity of the porous medium for packed beds of spherical particles as a function of the medium porosity $\phi$ and the thermal conductivity ratio between the fluid and the solid $\lambda$ can be expressed as in the following correlation $[4,8,20]$ :

$$
\frac{k_{s t}}{k}=(1-\sqrt{1-\phi})+\frac{2 \sqrt{1-\phi}}{1-\lambda \gamma}\left[\frac{(1-\lambda) \gamma}{(1-\lambda \gamma)^{2}} \ln \left(\frac{1}{\lambda \gamma}\right)-\frac{\gamma+1}{2}-\frac{\gamma-1}{1-\lambda \gamma}\right]
$$

Where $k$ is the thermal conductivity of the fluid, $\lambda=k / k_{s o}$ is the thermal conductivity ratio of the fluid phase to that of the solid phase, and the parameter $\gamma$ is a function of the porosity, which can be expressed by $[4,8,20]$ :

$$
\gamma=1.25[(1-\phi) / \phi]^{10 / 9}
$$

In the case of prevalent inertia effect, the thermal dispersion effect may become important. In order to examine the effect of thermal dispersion on heat transfer rate, the following thermal dispersion model will be used in the analysis $[4,8,21]$ :

$$
k_{s}=0.04\left(\rho_{\infty} c\right) u d_{p}(1-\phi) / \phi
$$

Next, the system of equations (1-5) together with the boundary conditions $(7 \& 8)$ will be transformed into a dimensionless form.

\section{Dimensionless variables.}

In order to obtain a system of equations applicable to the entire regime of mixed convection, the following dimensionless variables are introduced $[7,8,22]$ : 


$$
\begin{aligned}
& \eta=\frac{y}{x} P e_{x}^{1 / 2} \zeta^{-1}, \quad \zeta=\left[1+\frac{R a_{x}^{1 / 3}}{P e_{x}^{1 / 2}}\right]^{-1} \\
& f(\zeta, \eta)=\psi(x, y) /\left(\alpha P e_{x}^{1 / 2} \zeta^{-1}\right) \\
& \theta(\zeta, \eta)=\left(T-T_{\infty}\right) /\left[T_{w}(x)-T_{\infty}\right] \\
& \Phi(\zeta, \eta)=\left(C-C_{\infty}\right) /\left[C_{w}(x)-C_{\infty}\right]
\end{aligned}
$$

Where $\eta, f, \theta, \Phi$, and $\alpha$ are the pseudosimilarity variable, dimensionless stream function, dimensionless temperature, dimensionless concentration, and thermal diffusivity of fluid respectively. $\psi$ is the stream function, which is defined by $u=\partial \psi / \partial y$ and $v=-\partial \psi / \partial x$ such that the continuity equation, equation (1), is automatically satisfied; $P e_{x}=U_{\infty} x / \alpha$ is the local Peclet number, $R a_{x}=K_{\infty} g \beta_{T} x \Delta T \rho_{\infty} /(\alpha \mu)$ is the local Rayleigh number, and the parameter $\zeta$ is the nonsimilarity mixed-convection parameter. A value of $\zeta=0$ corresponds to pure free convection, while $\zeta=1$ represents pure forced convection. It will choose $\kappa=x \zeta / P e_{x}^{1 / 2}$ such that equation (9) and $\alpha_{e}$ are purely function of $\eta$ only [19]. By substituting equations (14) and (15) into equations (2), (4), and (5), the following nonsimilar system of dimensionless equations are obtained [22]:

10. Dimensionless momentum equation.

$$
\begin{aligned}
& {\left[1+A_{1} f^{\prime}\right] f^{\prime \prime}=A_{2} f^{\prime}-A_{3}\left(f^{\prime}\right)^{2}} \\
& -A_{4}\left\{n \theta+A_{5} \eta \theta^{\prime}+A_{6} \frac{\partial \theta}{\partial \zeta}+N\left[n \Phi+A_{5} \eta \Phi^{\prime}+A_{6} \frac{\partial \Phi}{\partial \zeta}\right]\right\}
\end{aligned}
$$

Where $N=\beta_{C}\left[C_{w}(x)-C_{\infty}\right] / \beta_{T}\left[T_{w}(x)-T_{\infty}\right]$ represents the buoyancy ratio, $A_{1} \rightarrow A_{6}$ are as follows:

$$
A_{1}=2\left(\frac{b}{b_{\infty}}\right) R\left(1+d e^{-\eta}\right)
$$

Where $R=\left(K_{\infty} b_{\infty} \alpha / v x\right)\left(P e_{x}^{1 / 2}+R a_{x}^{1 / 3}\right)^{2}$ is the porous medium inertia effect parameter.

$$
\begin{aligned}
& A_{2}=\frac{-d e^{-\eta}}{\left(1+d e^{-\eta}\right)} \quad A_{3}=R \frac{1.75 \phi_{\infty} d^{*} e^{-\eta}(3-2 \phi)\left(1+d e^{-\eta}\right)}{d_{p} \phi^{4} b_{\infty}} \\
& A_{4}=(1-\zeta)^{3}\left(1+d e^{-\eta}\right) \quad A_{5}=\frac{1}{3}\left[n(1-\zeta)-\frac{1}{2}(4-\zeta)\right] \quad A_{6}=\frac{1}{3}\left(\frac{1}{2}-n\right) \zeta(1-\zeta)
\end{aligned}
$$

11. Dimensionless energy equation.

$$
\begin{aligned}
& {\left[\frac{k_{s t}}{k}+B_{1} f^{\prime}\right] \theta^{\prime \prime}-\left\{B_{2}\left\{-\frac{1}{2}+B_{3}\left\{B_{4}\left\{B_{5}\right\}+B_{6} B_{7}\right\}\right\}-B_{8} f-B_{9}\left[B_{10} f^{\prime}+B_{11} f^{\prime \prime}\right]\right\} \theta^{\prime}} \\
& -n f^{\prime} \theta=B_{12}\left[\frac{\partial \theta}{\partial \zeta} f^{\prime}-\frac{\partial f}{\partial \zeta} \theta^{\prime}\right]
\end{aligned}
$$


Where $B_{1} \rightarrow B_{12}$ are as follows:

$B_{1}=0.04 D_{s} \frac{(1-\phi)}{\phi}$

Where $D_{s}=\left(d_{p} / x\right)\left(P e_{x}^{1 / 2}+R a_{x}^{1 / 3}\right)^{2}$ is the porous medium thermal dispersion parameter.

$$
\begin{aligned}
& B_{2}=\frac{-\phi_{\infty} d^{*} e^{-\eta}}{(1-\phi)^{1 / 2}} \quad B_{3}=\frac{1}{(1-\lambda \gamma)} \quad B_{4}=\left(\frac{25}{9}\right) \frac{(1-\phi)}{\phi^{2}(1-\lambda \gamma)^{2}}\left[\frac{(1-\phi)}{\phi}\right]^{1 / 9} \\
& B_{5}=(1-\lambda)\left[\frac{1+\lambda \gamma}{1-\lambda \gamma} \ln \left(\frac{1}{\lambda \gamma}\right)-2\right]-\frac{1}{2}(1-\lambda \gamma)^{2} \quad B_{6}=\left[1+\frac{\left.\left(\frac{25}{9}\right) \lambda(1-\phi)\left[\frac{(1-\phi)}{\phi}\right]^{1 / 9}\right]}{\phi^{2}(1-\lambda \gamma)}\right] \\
& B_{7}=\left[\frac{(1-\lambda) \gamma}{(1-\lambda \gamma)^{2}} \ln \left(\frac{1}{\lambda \gamma}\right)-\frac{\gamma+1}{2}-\frac{\gamma-1}{1-\lambda \gamma}\right] \quad B_{8}=\frac{1}{3}\left[\left(n-\frac{1}{2}\right)(1-\zeta)+\frac{3}{2}\right] \quad B_{9}=0.04 D_{s} \\
& B_{10}=\frac{\phi_{\infty} d^{*} e^{-\eta}}{\phi^{2}} \\
& B_{11}=\frac{(1-\phi)}{\phi}
\end{aligned}
$$

12. Dimensionless concentration equation.

$$
\frac{1}{L e} \Phi^{\prime \prime}+B_{8} f \Phi^{\prime}-n f \Phi=A_{6}\left[f^{\prime} \frac{\partial \Phi}{\partial \zeta}-\frac{\partial f}{\partial \zeta} \Phi^{\prime}\right]
$$

Where $L e=\alpha / D$ is the Lewis number.

13. Dimensionless boundary conditions.

$$
\begin{aligned}
& \left\{B_{8} f(\zeta, 0)+A_{6} \frac{\partial f(\zeta, 0)}{\partial \zeta}\right\}=f_{w} \\
& \theta(\zeta, 0)=1 \\
& \Phi(\zeta, 0)=1
\end{aligned}
$$

Where $f_{w}=\left(-V_{w} x\right) /\left[\alpha\left(P e_{x}^{1 / 2}+R a_{x}^{1 / 3}\right)\right]$ is the surface mass flux parameter. $f_{w}$ increases from injection to suction; i.e. from -1 to 1 .

$$
\begin{aligned}
& f^{\prime}(\zeta, \infty)=\zeta^{2} \\
& \theta(\zeta, \infty)=0 \\
& \Phi(\zeta, \infty)=0
\end{aligned}
$$

Physical quantities of interest include the velocity components $u$ and $v$ in the $x$ and $y$ directions, the local Nusselt number $N u_{x}=h x / k$, where the local heat transfer coefficient $h=q_{w} /\left[T_{w}(x)-T_{\infty}\right]$ and $q_{w}=-k_{e}(\partial T / \partial y)_{y=0}$, the local Sherwood number $S h_{x}=h_{m} x / D$, where the local mass transfer coefficient $h_{m}=m_{w} /\left[C_{w}(x)-C_{\infty}\right]$ and $m_{w}=-D(\partial C / \partial y)_{y=0}$, the local surface shear stress $\tau_{w}$, defined as $\tau_{w}=\mu(\partial u / \partial y)_{y=0}$. In terms of the new variables, these quantities have the expressions: 


$$
\begin{aligned}
& u=\left(U_{\infty} / \zeta^{2}\right) f^{\prime} \\
& v=-\frac{\alpha}{x} P e_{x}^{1 / 2} \frac{1}{\zeta}\left\{B_{8} f+A_{5} \eta f^{\prime}+A_{6} \frac{\partial f}{\partial \zeta}\right\} \\
& \frac{N u_{x}}{P e_{x}^{1 / 2} \zeta^{-1}}=-\left[\frac{k_{s t}}{k}+B_{1} f^{\prime}(\zeta, 0)\right] \theta^{\prime}(\zeta, 0) \\
& \frac{S h_{x}}{P e_{x}^{1 / 2} \zeta^{-1}}=-\Phi^{\prime}(\zeta, 0) \\
& \tau_{w} \frac{x^{2}}{\mu \alpha\left(P e_{x}^{1 / 2}+R a_{x}^{1 / 3}\right)^{3}}=f^{\prime \prime}(\zeta, 0)
\end{aligned}
$$

The primes in equations (16-25) denote partial differentiation with respect to $\eta$. The presence of $\partial / \partial \zeta$ in these equations makes them nonsimilar [9].

\section{Numerical scheme}

The numerical scheme to solve equations (16-18) adopted here is based on a combination of the following concepts [23]:

1. The boundary conditions for $\eta=\infty$ are replaced by $f^{\prime}\left(\zeta, \eta_{\max }\right)=\zeta^{2}, \theta\left(\zeta, \eta_{\max }\right)=0$, and $\Phi\left(\zeta, \eta_{\max }\right)=0$ where $\eta_{\max }$ is a sufficiently large value of $\eta$ where the boundary conditions (20) for velocity is satisfied.

2. The two-dimensional domain of interest $(\zeta, \eta)$ is discretized with an equispaced mesh in the $\zeta$ direction and another equispaced mesh in the $\eta$ direction.

3. The partial derivatives with respect to $\zeta$ and $\eta$ are all evaluated by the central difference approximations. The central difference approximation for the partial derivatives with respect to $\zeta$ vanish when $\zeta=0$ and $\zeta=1$.

4. Two iteration loops based on the successive substitution are used because of the nonlinearity of the equations.

5.In each inner iteration loop, the value of $\zeta$ is fixed, while each of equations (16-18) is solved as a linear second-order boundary-value problem of ordinary differential equation (ODE) on the $\eta$ domain. The inner integration is continued until the nonlinear solution converges for the fixed value of $\zeta$.

6. In the outer iteration loop, the value of $\zeta$ is advanced from 0.1 to 0.9. The derivatives with respect to $\zeta$ are updated after every outer iteration step.

Fortran language is used to program the system of nonlinear equations. The program is divided into three parts: the first part obtains the solution of the pure free convection by setting the nonsimilarity parameter equal to zero. The second part obtains the solution of pure forced convection by setting the nonsimilarity parameter equal to one. The third part obtains the solution of mixed convection region for the nonsimilarity parameter values lies between 0 and 1 (i.e., $0<\zeta<1$ ).

In this work $\phi_{\infty}=0.39, d_{p}=0.005 \mathrm{~m}, \lambda=1$ [4], and step sizes of $\Delta \eta=0.02$ and $\Delta \zeta=0.1$ are input to the program. A convergence criterion of $\sum_{j=1}^{N_{n}}\left|f_{\text {new }}(j, i)-f_{\text {old }}(j, i)\right|<$ 0.001 is adopted in the program for all types of convection. Where $f_{\text {new }}^{j=1}(j, i)$ and $f_{\text {old }}(j, i)$ are the new and old value of $f$. For the mixed convection region, as well as the above 
mentioned convergence criterion there is another convergence criterion on the whole region of mixed convection that it

$$
\begin{aligned}
& \text { For } i=1, \ldots, N_{\zeta} \\
& \sum_{N_{n}}\left|f_{\text {new }}(j, i)-f_{\text {na }}(j, i)\right|<0.001
\end{aligned}
$$

If (yes) ${ }^{j}$ then next $i$. If (no) then updates the values of $f$ 's, $\theta$ 's, and $\Phi$ 's for the mixed convection region $(0<\zeta<1)$ and repeat the solution process for this region. Where $f_{n a}(j, i)$ represent the assumed value of $f$ that is not affected by the first convergence criterion.

\section{Results and discussion}

Numerical results were obtained and presented. These results cover the values of the exponent $n$, which is physically realistic. The range of $n$ values for which the present problem is physically realistic can be found following the criterion given by Aldoss et al. [7] and Chen [8]. The criterion is based on the requirements that both $u$ and $\delta_{T}$, must increase or at least remain constant with respect to $x$ as long as the wall temperature at $x>0$ is different from that of the surroundings. To meet these requirements, it is found that $\delta_{T}$ varies like $x^{(2-n) / 3}$ and $u$ varies like $x^{(2 / 3)[n-(1 / 2)]}$ for pure free convection. Thus, the range of exponent $n$ is $0.5 \leq n \leq 2$. In order to illustrate the effect of all involved parameters on the local Nusselt and Sherwood numbers a parametric study is performed. Table (1) shows the input values of the parameters to the program.

Table (1): Input values of the parameters to the program when

\begin{tabular}{|c|c|c|c|c|c|c|}
\hline Run number & $f_{w}$ & $n$ & $R$ & $N$ & $D_{s}$ & Le \\
\hline 1 & 0 & 0.5 & 0 & 0 & 0 & 1 \\
\hline 2 & 0 & 1 & 0 & 0 & 0 & 1 \\
\hline 3 & 0 & 1.5 & 0 & 0 & 0 & 1 \\
\hline 4 & 0 & 2 & 0 & 0 & 0 & 1 \\
\hline 5 & 0 & 0.5 & 0 & 5 & 0 & 10 \\
\hline 6 & 0 & 1 & 0 & 5 & 0 & 10 \\
\hline 7 & 0 & 1.5 & 0 & 5 & 0 & 10 \\
\hline 8 & 0 & 2 & 0 & 5 & 0 & 10 \\
\hline 9 & 0 & 1 & 0 & 2 & 0 & 10 \\
\hline 10 & 0 & 1 & 0.1 & 2 & 0 & 10 \\
\hline 11 & 0 & 1 & 1 & 2 & 0 & 10 \\
\hline 12 & 0 & 1 & 10 & 2 & 0 & 10 \\
\hline 13 & 0 & 1 & 1 & 2 & 15 & 10 \\
\hline 14 & 0 & 1 & 1 & 2 & 20 & 10 \\
\hline 15 & 0 & 1 & 1 & 2 & 25 & 10 \\
\hline 16 & 0 & 1 & 1 & 2 & 30 & 10 \\
\hline 17 & 0 & 1 & 0 & 1 & 0 & 0.5 \\
\hline 18 & 0 & 1 & 0 & 1 & 0 & 5 \\
\hline 19 & 0 & 1 & 0 & 1 & 0 & 10 \\
\hline 20 & 0 & 1 & 0 & 1 & 0 & 100 \\
\hline 21 & 0 & 1 & 0 & 0 & 0 & 10 \\
\hline 22 & 0 & 1 & 0 & 1 & 0 & 10 \\
\hline 23 & 0 & 1 & 0 & 5 & 0 & 10 \\
\hline 24 & 0 & 1 & 0 & 8 & 0 & 10 \\
\hline 25 & -1 & 1 & 0 & 1 & 0 & 0.5 \\
\hline 26 & -0.5 & 1 & 0 & 1 & 0 & 0.5 \\
\hline 27 & 0 & 1 & 0 & 1 & 0 & 0.5 \\
\hline 28 & 0.5 & 1 & 0 & 1 & 0 & 0.5 \\
\hline 29 & 1 & 1 & 0 & 1 & 0 & 0.5 \\
\hline
\end{tabular}
$\left(\phi_{\infty}=0.39, d_{p}=0.005 \mathrm{~m}, \lambda=1, \Delta \eta=0.02, \Delta \zeta=0.1\right)$. 

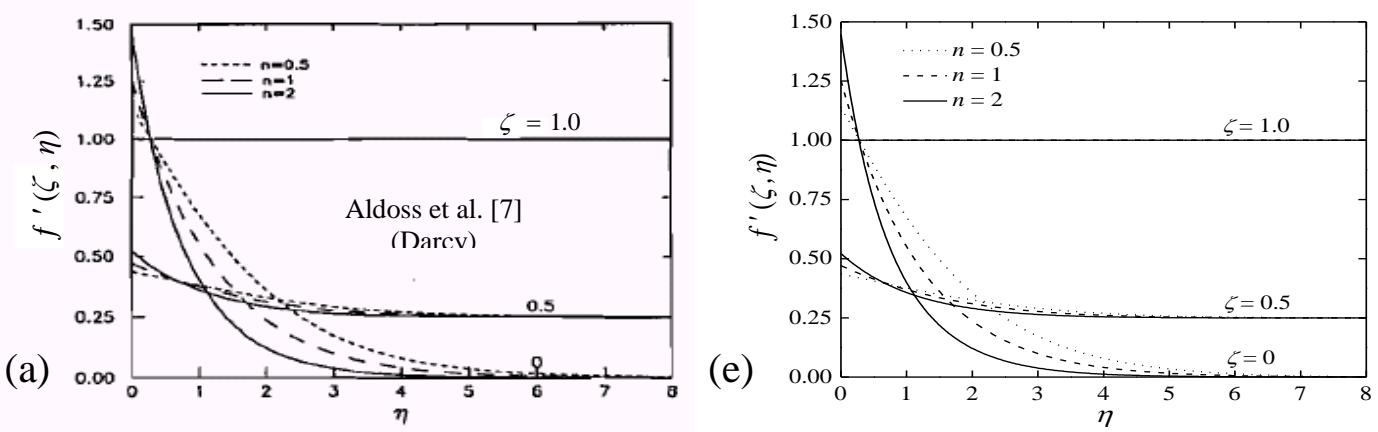

(b)
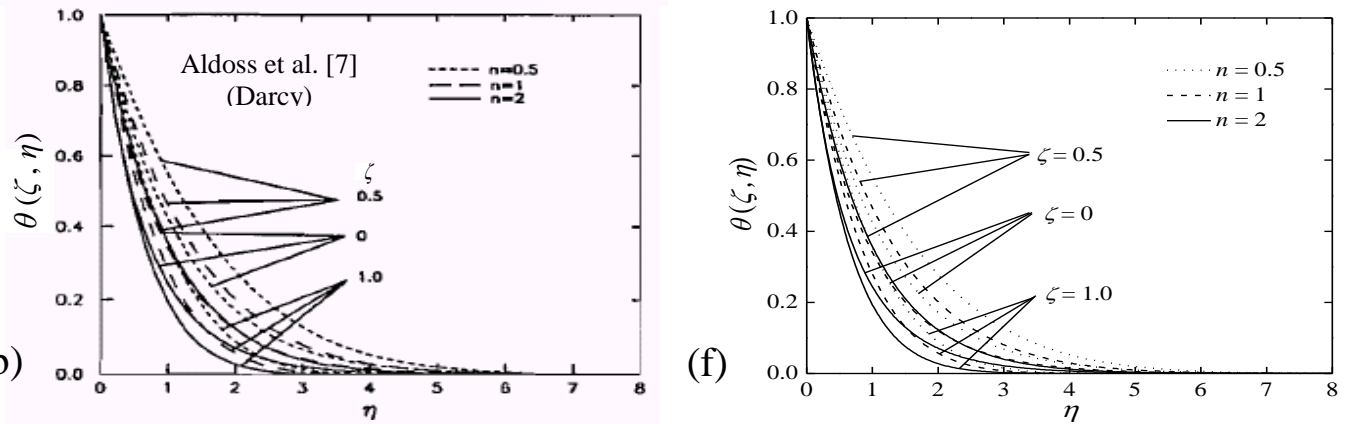

(c)
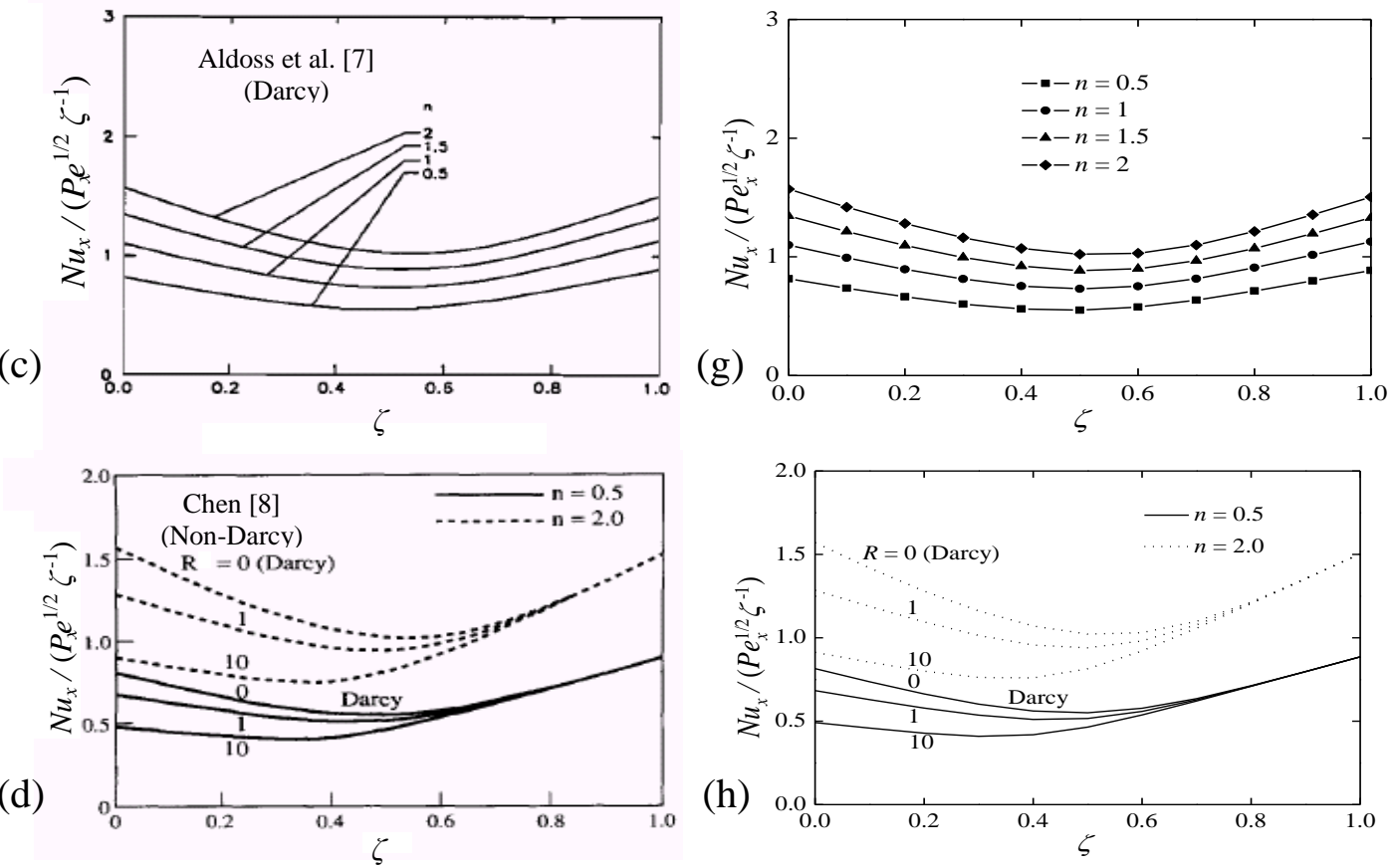

Previous work

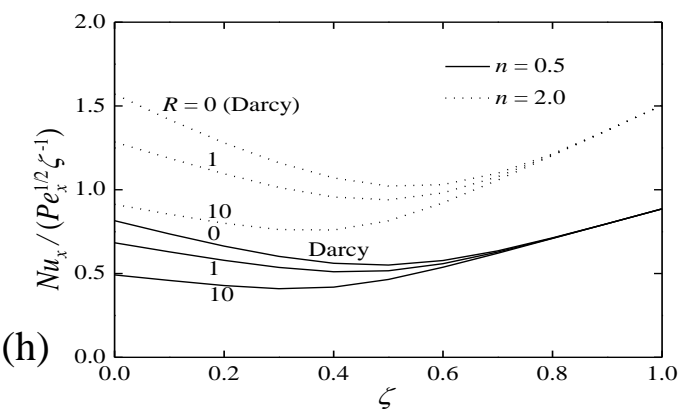

Present work

Figure (2): Comparisons of velocity profile, temperature profile, and local Nusselt number for Darcy and non-Darcy solution.

The whole runs are achieved two times one under uniform permeability (UP) condition when $d=d^{*}=0$, while the other under variable permeability (VP) condition when $d=3$ and $d^{*}=1.5$. The results have been obtained for various values of the parameters. In order to validate the numerical results, the present results are compared with those of previously published works on special cases of the problem. These favorable comparisons give confidence in the numerical results to be reported in the next sections. Comparisons of 
velocity profile, temperature profile, and local Nusselt number for Darcy and non-Darcy solution are shown graphically in Figure (2). In tabulated form, comparisons of $N u_{x} /\left(P e_{x}^{1 / 2} \zeta^{-1}\right)$ values for Darcy solution at values of $\zeta$ and $n$ are listed in Table (2). These comparisons were found to be in excellent agreement

Table (2): Comparisons of $N u_{x} /\left(P e_{x}^{1 / 2} \zeta^{-1}\right)$ values for Darcy solution at values of $\zeta$ and $n$.

\begin{tabular}{|c|c|c|c|c|}
\hline$\zeta$ & $n=0.5$ & $n=1.0$ & $n=1.5$ & $n=2.0$ \\
\hline 0.0 & 0.8165 & 1.0995 & 1.3458 & 1.5710 \\
\hline 0.1 & 0.7373 & 0.9930 & 1.2156 & 1.4191 \\
\hline 0.2 & 0.6648 & 0.8959 & 1.0970 & 1.2808 \\
\hline 0.3 & 0.6036 & 0.8134 & 0.9956 & 1.1613 \\
\hline 0.4 & 0.5619 & 0.7547 & 0.9208 & 1.0708 \\
\hline 0.5 & 0.5510 & 0.7313 & 0.8852 & 1.0231 \\
\hline 0.6 & 0.5775 & 0.7523 & 0.9002 & 1.0314 \\
\hline 0.7 & 0.6356 & 0.8160 & 0.9670 & 1.0997 \\
\hline 0.8 & 0.7126 & 0.9089 & 1.0722 & 1.2148 \\
\hline 0.9 & 0.7980 & 1.0162 & 1.1973 & 1.3552 \\
\hline 1.0 & 0.8863 & 1.1285 & 1.3294 & 1.5046 \\
\hline
\end{tabular}

(a) Aldoss et al. [7]

\begin{tabular}{|c|c|c|c|c|}
\hline$\zeta$ & $n=0.5$ & $n=1.0$ & $n=1.5$ & $n=2.0$ \\
\hline 0.0 & 0.8156 & 1.0990 & 1.3456 & 1.5170 \\
\hline 0.1 & 0.7361 & 0.9922 & 1.2153 & 1.4191 \\
\hline 0.2 & 0.6635 & 0.8950 & 1.0966 & 1.2806 \\
\hline 0.3 & 0.6025 & 0.8127 & 0.9951 & 1.1641 \\
\hline 0.4 & 0.5614 & 0.7543 & 0.9204 & 1.0706 \\
\hline 0.5 & 0.5509 & 0.7311 & 0.8850 & 1.0229 \\
\hline 0.6 & 0.5775 & 0.7523 & 0.9002 & 1.0314 \\
\hline 0.7 & 0.6356 & 0.8160 & 0.9670 & 1.0998 \\
\hline 0.8 & 0.7125 & 0.9089 & 1.0722 & 1.2148 \\
\hline 0.9 & 0.7980 & 1.0161 & 1.1973 & 1.3552 \\
\hline 1.0 & 0.8862 & 1.1284 & 1.3293 & 1.5045 \\
\hline
\end{tabular}

(b) Chen [8]

\begin{tabular}{|c|c|c|c|c|}
\hline$\zeta$ & $n=0.5$ & $n=1.0$ & $n=1.5$ & $n=2.0$ \\
\hline 0.0 & 0.8158 & 1.0989 & 1.3452 & 1.5702 \\
\hline 0.1 & 0.7361 & 0.9921 & 1.215 & 1.4186 \\
\hline 0.2 & 0.6635 & 0.8951 & 1.0966 & 1.2805 \\
\hline 0.3 & 0.6026 & 0.8128 & 0.9953 & 1.1612 \\
\hline 0.4 & 0.5615 & 0.7542 & 0.9203 & 1.0705 \\
\hline 0.5 & 0.5508 & 0.7307 & 0.8845 & 1.0223 \\
\hline 0.6 & 0.5774 & 0.7519 & 0.8996 & 1.0307 \\
\hline 0.7 & 0.6355 & 0.8158 & 0.9667 & 1.0993 \\
\hline 0.8 & 0.7125 & 0.9088 & 1.072 & 1.2146 \\
\hline 0.9 & 0.7979 & 1.0161 & 1.1972 & 1.3549 \\
\hline 1.0 & 0.8862 & 1.1281 & 1.329 & 1.5041 \\
\hline
\end{tabular}

(c) Present work 


\section{Results under UP condition:}

In this section the results under uniform permeability condition for different parameters will be presented and discussed. These parameters include power law index $n$, inertia effect parameter $R$, thermal dispersion parameter $D_{s}$, Lewis number $L e$, buoyancy ratio $N$, and surface mass flux parameter $f_{w}$. To conserve space, the variations of velocity, temperature, and concentration profiles with pseudosimilarity variable at selected values of the nonsimilarity mixed convection parameter and any specified parameter will be described only and not shown graphically.

\section{Effect of exponent $n$ :}

At a given value of $n$ the velocity gradient at the surface is found to be greater at lower values of $\zeta$. At a given value of $\zeta$, as $n$ increases the velocity gradient is larger and the momentum boundary layer thickness is smaller. It is noticed that at aiven value of $n$, as $\zeta$ increases the thermal and concentration boundary layer thickness increases, while the temperature and concentration gradients at the surface decreases. This continues to be true until a certain value of $\zeta$ is reached, beyond which as $\zeta$ increases further, the trend starts to reverse, and the thermal and concentration boundary layer thickness decreases while the temperature and concentration gradients at the surface increases. At a fixed value of $\zeta$, as $n$ increases the thermal and concentration boundary layer thickness decreases.

The variations of local Nusselt and Sherwood numbers with $\zeta$ at values of $n$ are illustrated in Figure (3). At a given value of $n$ as $\zeta$ increases from 0 the local Nusselt and Sherwood numbers decreases, reaches a minimum value at a certain value of $\zeta$, and then increases again as $\zeta$ approaches 1 . It is also clear that for a higher value of $n$, the local Nusselt and Sherwood numbers is larger. For all values of $n$ the local Nusselt and Sherwood numbers for pure free convection at $\zeta=0$ is higher than that of pure forced convection at $\zeta=1$.

(a)

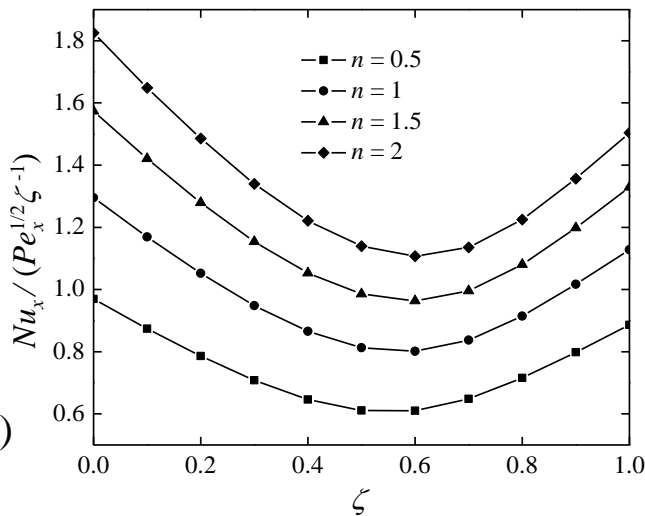

(b)

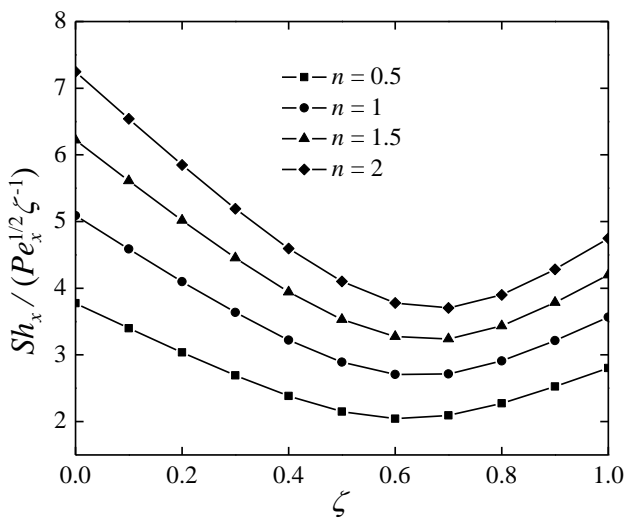

Figure (3): Effect of exponent $n$ (UP).

The behavior of the curves with a minimum value at a certain $\zeta$ value is due to the definition of the local Nusselt and Sherwood numbers Parameters and does not indicate that the $N u_{x}$ and $S h_{x}$ values for mixed convection is smaller than that for pure free and forced convection. For example, for $n=1$ and at $\zeta=0.5$, with $P e_{x}=10^{2}$ and $R a_{x}=10^{3}$, one finds 
$N u_{x}=16.262$ as compared with $N u_{x}=12.951$ for pure free convection at $\zeta=0$ and $N u_{x}=11.281$ for pure forced convection at $\zeta=1$.

\section{Effect of inertia parameter $R$ :}

At a given value of $\zeta$ an increase in the parameter $R$ reduces the fluid velocity. However, at $\zeta=0$ the velocity profiles thin near the surface, while it thick far from the surface as the parameter $R$ increases. Also, at a given value of $\zeta$ an increase in the parameter $R$ increases the fluid temperature and concentration. When $\zeta=1$ increasing of $R$ has no effect on the temperature and concentration profiles.

The variations of local Nusselt and Sherwood numbers with $\zeta$ at values of $R$ are given in Figure (4). The local Nusselt and Sherwood numbers decreases with an increase in $R$. This is evident from the fact that inertia effect tends to retard the momentum transport in the boundary layer and to reduce the heat and mass transfer. Also, the inertia term has a pronounced effect on the heat and mass transfer rates for higher values of $R$. Moreover, all curves corresponding to different values of $R$ are seen to converge to one point in the forced convection limit. This implies that the inertia term has little significance in forced convection when the Ergun's correlation is used.

(a)

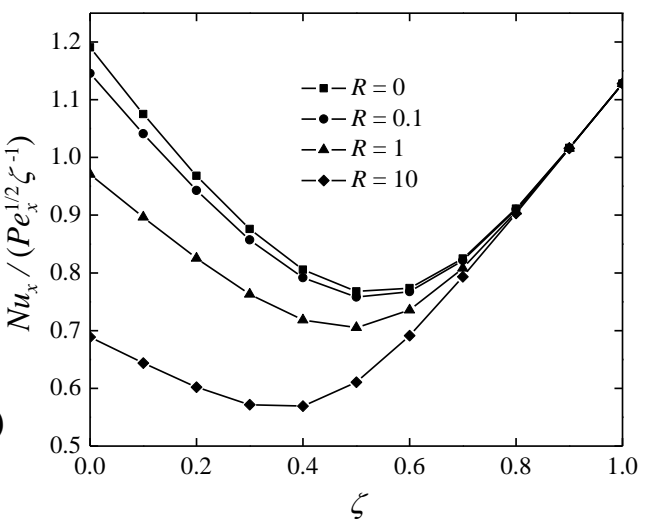

(b)

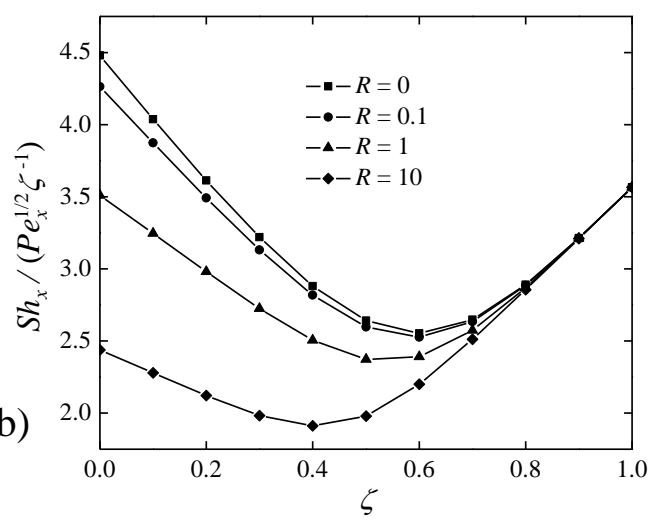

Figure (4): Effect of inertia parameter $R$ (UP).

\section{Effect of thermal dispersion parameter $D_{s}$ :}

At a given value of $\zeta$ both momentum and thermal boundary layer thickness increases as $D_{s}$ increases, while concentration boundary layer thickness decreases slightly. However, as $\zeta$ goes to 1 this effect on concentration profile is diminished until it is neglected when $\zeta=1$. Since the value of

$f^{\prime}(\zeta, 0)$ is always positive, it can be noticed from equation (23) that dispersion always enhances the heat transfer coefficient as shown in Figure $(5$ a). Starting from $\zeta=0$, it is clear that, increasing of $D_{s}$ leads to a small increase in the local Sherwood number, and as $\zeta$ increases to 1 this small increase diminished gradually until it is neglected as presented in Figure $(5 \mathrm{~b})$. 

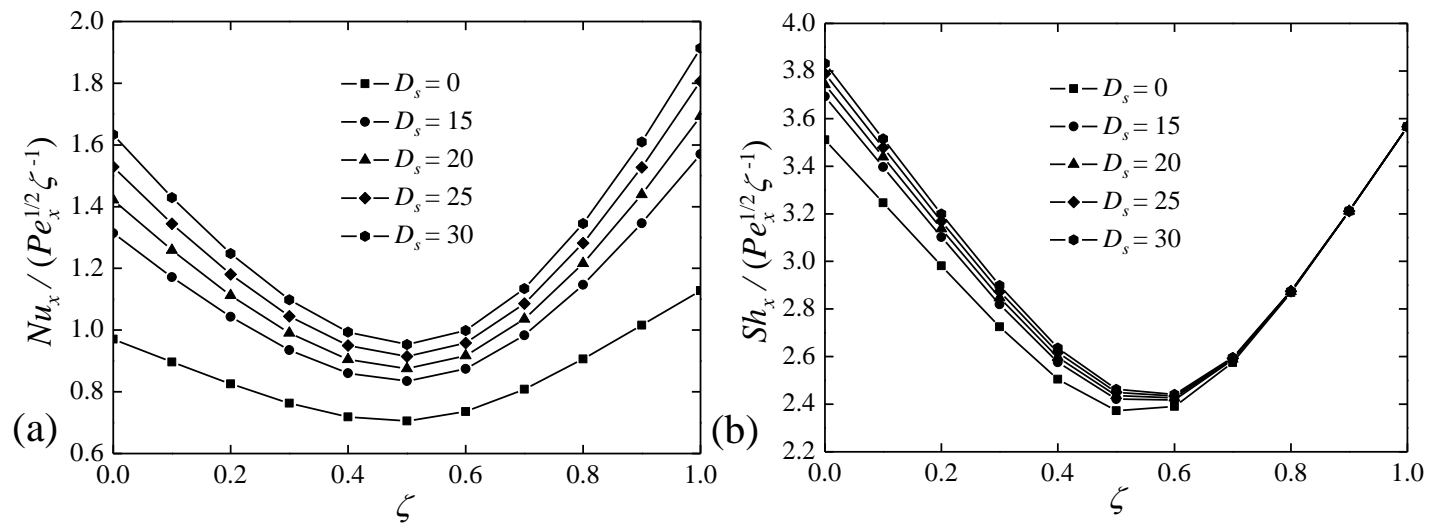

Figure (5): Effect of thermal dispersion parameter $D_{s}$ (UP).

\section{Effect of Lewis number $L e$ :}

Increases in the values of $L e$ resulted in decreases in the mass diffusivity. This, in turn, results in decrease in the concentration buoyancy forces and therefore the flow velocity. Also, increases in the values of $L e$ result in increases in the thermal boundary layer thickness. However, for larger values of $L e$ the decrease in the flow velocity and the increase in the fluid temperature are small. When, $\zeta=1$ the increase in $L e$ value has no effect on the thermal boundary layer thickness. As expected, increasing the value of $L e$ produces lower concentrations. Due to the above reasons the local Nusselt number decreases and the local Sherwood number increases as the value of $L e$ increases as depicted in Figure (6). Furthermore, in Figure ( 6 a) all curves corresponding to different values of $L e$ are seen to converge to one point in the forced convection limit.
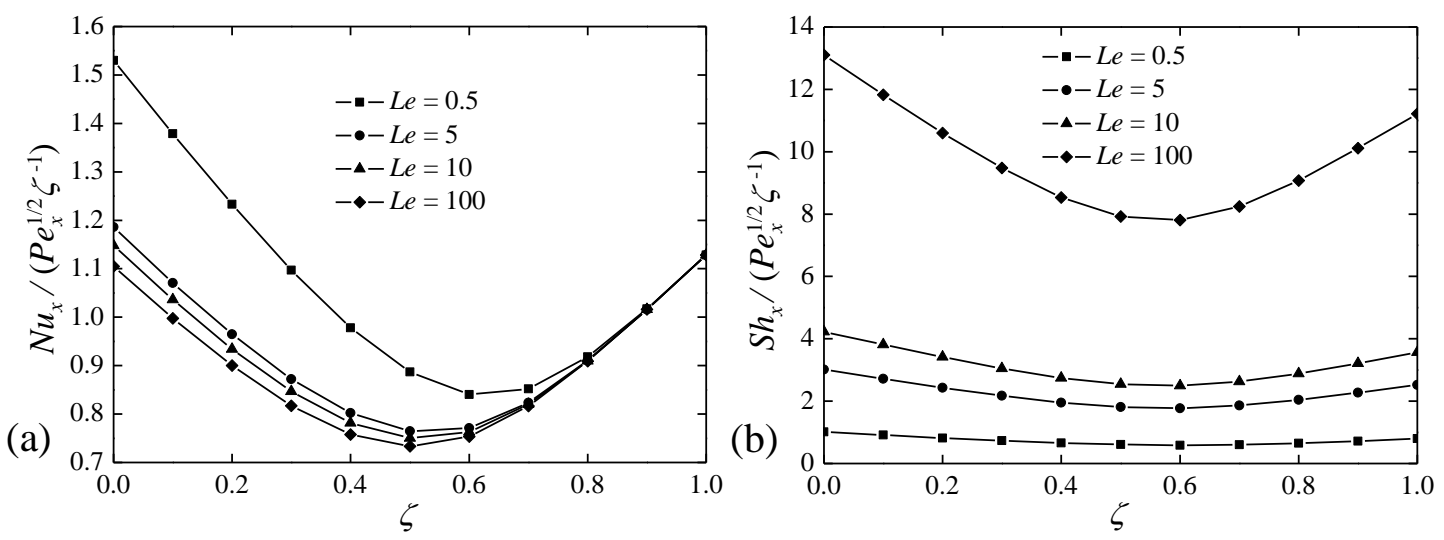

Figure (6): Effect of Lewis number $L e(\mathrm{UP})$.

\section{Effect of buoyancy ratio $N$ :}

Increasing the buoyancy ratio parameter results in increase of the buoyancy effect, causing higher flow velocities near the horizontal flat plate. The high velocity near the surface will carry more heat and mass out of the surface, thus decreases the thermal and concentration boundary layer thickness. This causes increases in the temperature and concentration gradients at the surface, which produces increases in the heat and mass transfer rates as shown in Figure (7). Also, from Figure (7), it can be seen that all curves 
corresponding to different $N$ values are converge to one point at $\zeta=1$. This means that the buoyancy ratio has large influence on free convection.
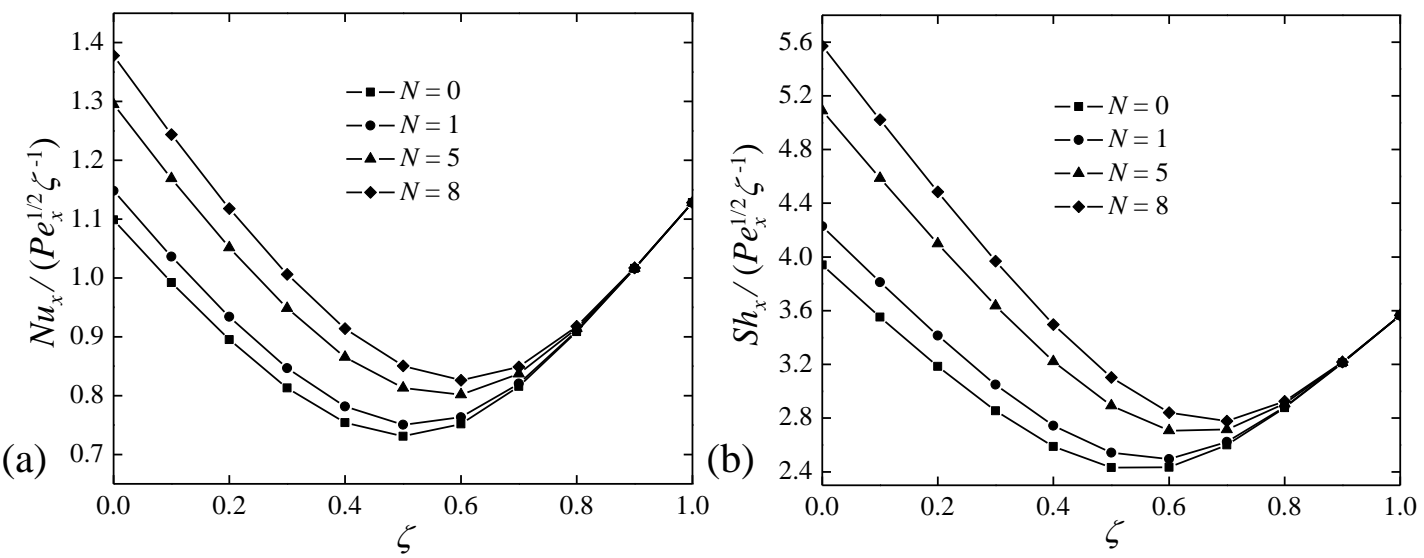

Figure (7): Effect of buoyancy ratio $N$ (UP).

\section{Effect of surface mass flux parameter $f_{w}$ :}

Surface fluid suction $\left(f_{w}>0\right)$ has the effect of reducing the velocity, temperature, and concentration boundary layer thickness. Consequently, the rate of heat and mass transfer increases. On the other hand, surface fluid injection or blowing $\left(f_{w}<0\right)$ produces the opposite effect, namely, a decrease in the surface heat and mass transfer rates. The behaviors of local Nusselt and Sherwood numbers are illustrated in the Figure (8), as the surface mass flux parameter $f_{w}$ moves from injection domain to suction domain.

(a)

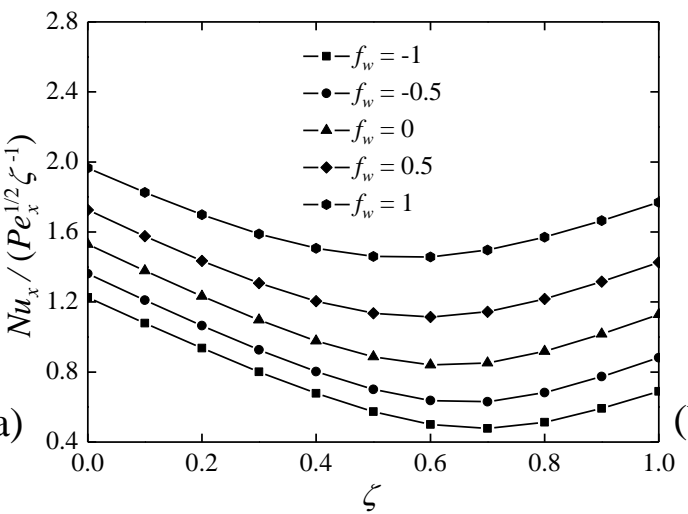

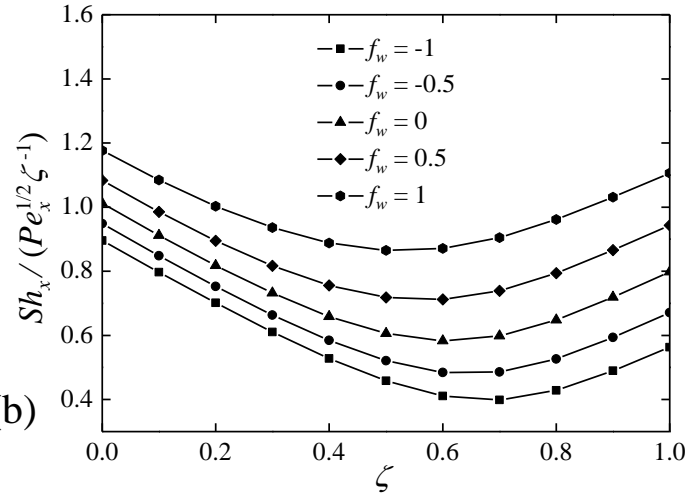

Figure (8): Effect of surface mass flux parameter $f_{w}$ (UP).

\section{Results under VP condition:}

Figures (9-14) presents the influence of the various parameters under variable permeability condition on the heat and mass transfer rates. For any particular parameter, variable permeability effect increase the velocity and reduce the thermal and concentration boundary layers in comparison with its counterpart for the case of uniform permeability. This leading is to an enhancement of heat and mass transfer rates. Starting from $\zeta=0$, as the inertia effect parameter increases, the momentum boundary layer thickness decreases while the thermal and concentration boundary layer thickness increases. This continues to be true until a certain value of $\zeta$ is reached, beyond which as $\zeta$ increases further the momentum 
boundary layer thickness increases while the thermal and concentration boundary layer thickness decreases. Due to the above reason, as $R$ increases the curves of local Nusselt and Sherwood numbers decreases and then they increases in the form illustrated in Figure (10).

(a)

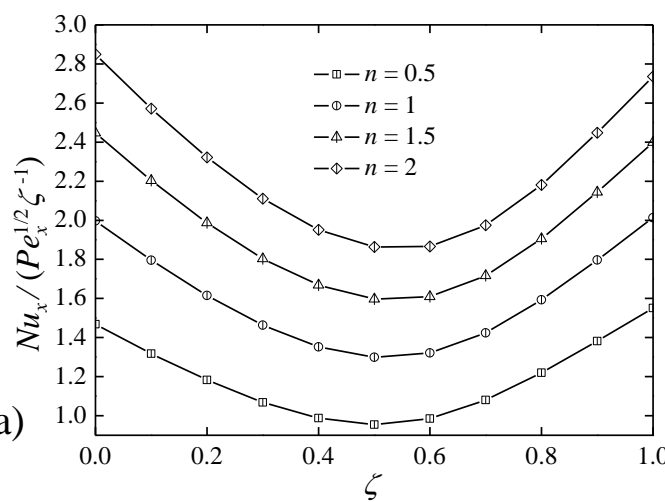

(b)

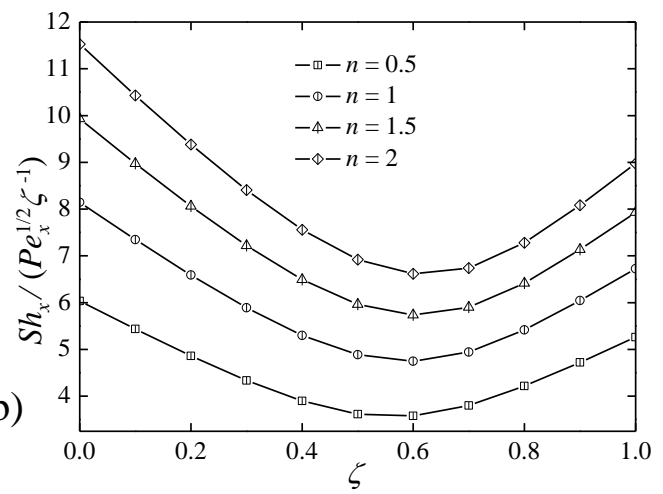

Figure (9): Effect of exponent $n$ (VP).
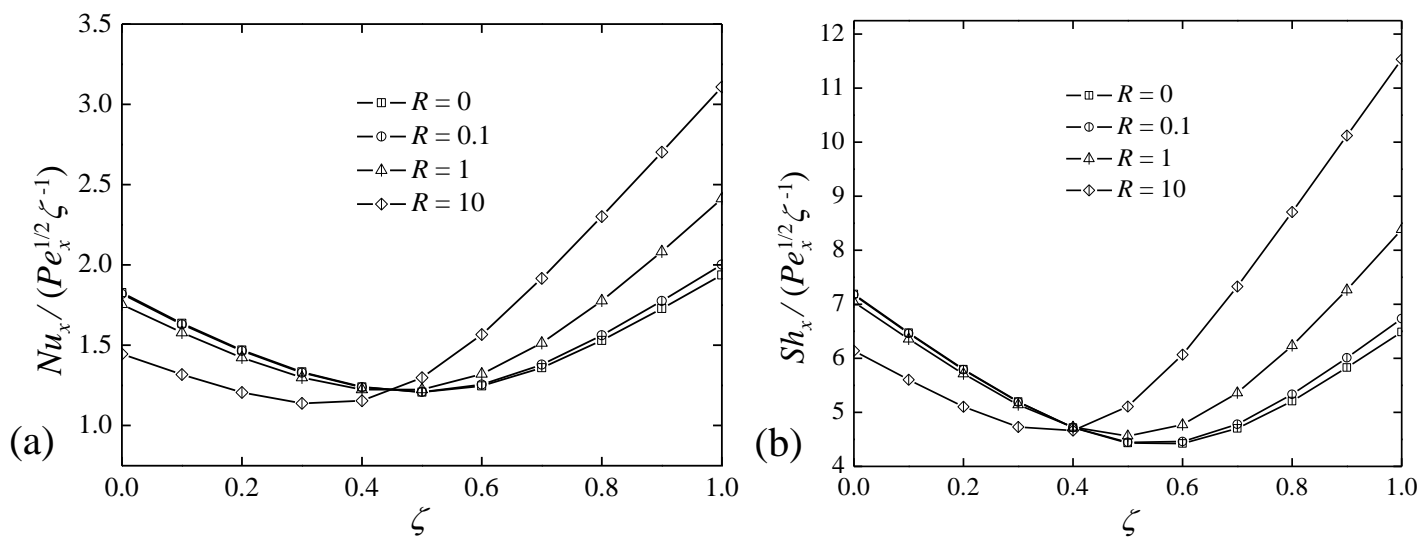

Figure (10): Effect of inertia parameter $R$ (VP).

(a)

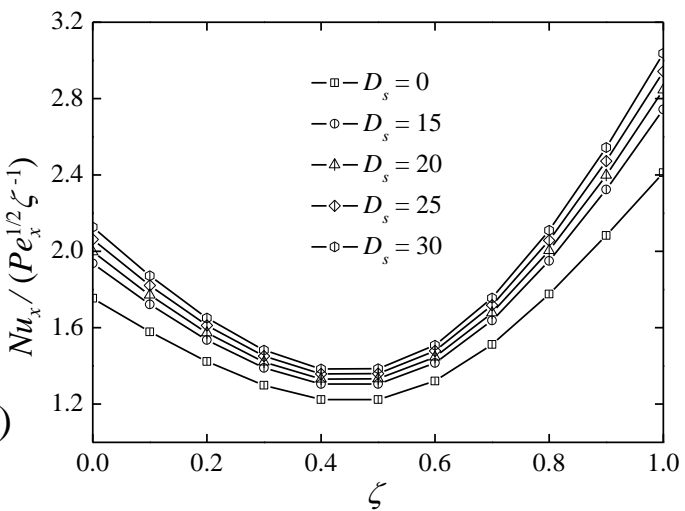

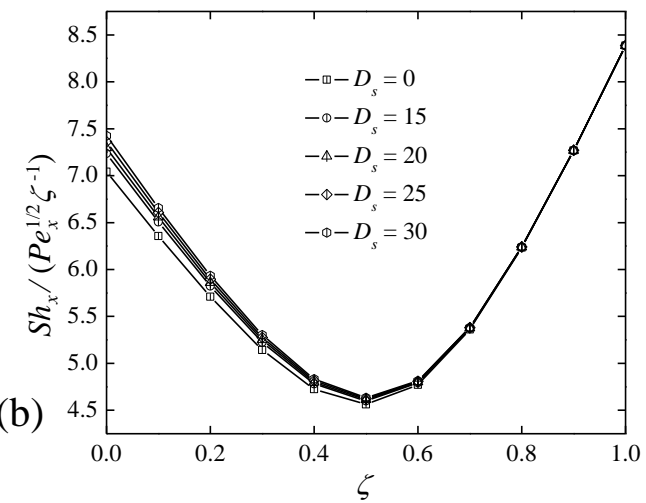

Figure (11): Effect of thermal dispersion parameter $D_{s}$ (VP). 
(a)

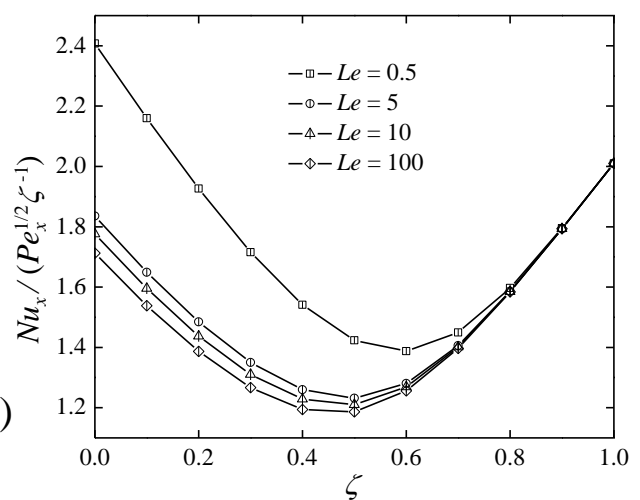

(b)

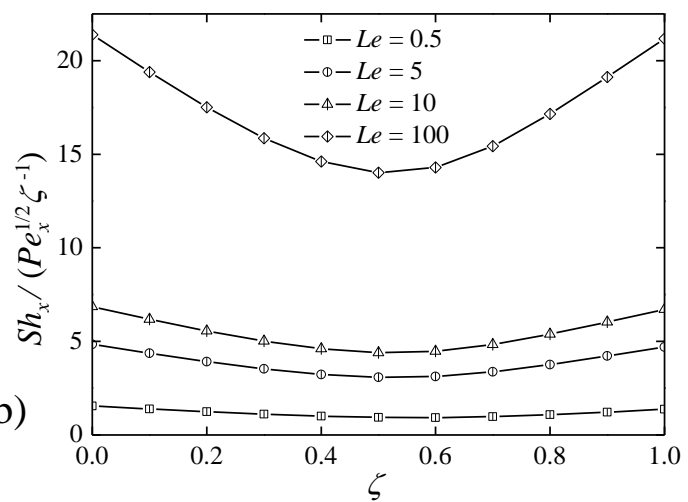

Figure (12): Effect of Lewis number Le (VP).

(a)

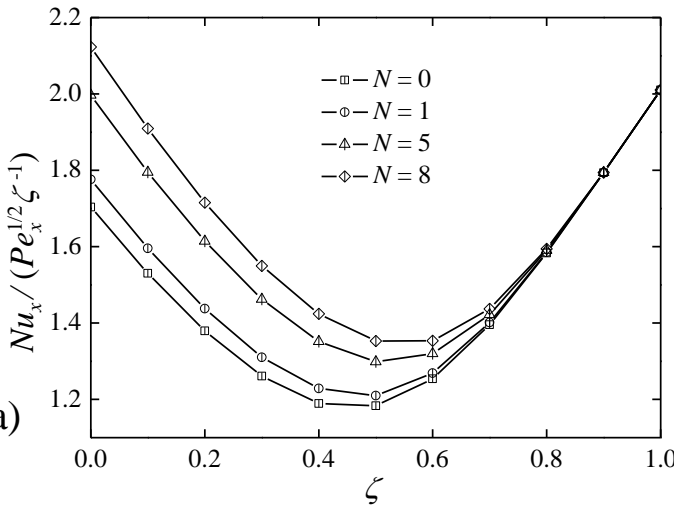

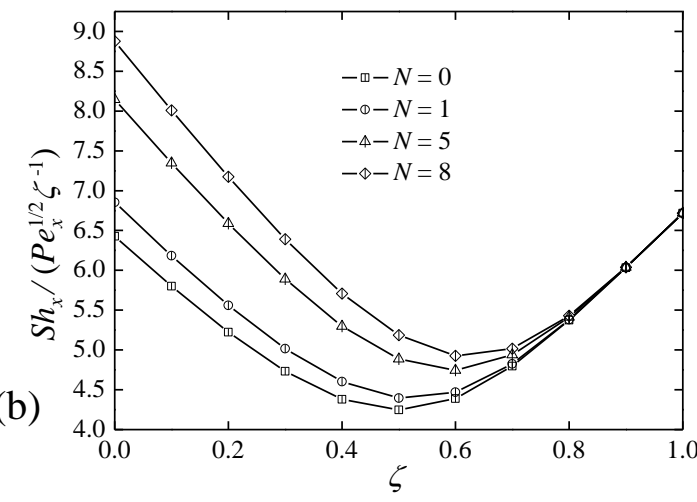

Figure (13): Effect of buoyancy ratio $N$ (VP).

\section{Conclusions}

The results indicated that under uniform permeability condition as the power law index for surface temperature and concentration increases, the local Nusselt and Sherwood numbers are increases. The local Nusselt and Sherwood numbers decreases with an increase in inertia effect parameter. Increasing the thermal dispersion parameter enhances the heat transfer rate and leads to a small increase in the mass transfer rate. The local Nusselt number decreases and the local Sherwood

number increases as the value of Lewis number increases. Increasing the buoyancy ratio parameter increases the heat and mass transfer rates. Surface fluid suction has the effect of increasing the rates of heat and mass transfer. Surface fluid injection has the opposite effect. For any particular parameter, variable permeability leads to an enhancement of heat and mass transfer rates.

\section{References}

1. Bera, P., and Khalili, A., "Stability of Mixed Convection in an Anisotropic Vertical Porous Channel," Physics of Fluids, Vol. 14, No. 5, PP. 1617-1630, May 2002.

2. Bejan, A., Convection Heat Transfer, Second Edition, John Wiley \& Sons, Inc., 1995. 
3. Chamkha, A. J., and Khaled, A.-R. A., "Nonsimilar Hydromagnetic Simultaneous Heat and Mass Transfer by Mixed Convection From a Vertical Plate Embedded in a Uniform Porous Medium," Numerical Heat Transfer, Part A, Vol. 36, PP. 327-344, 1999.

4. Chen, C.-H., "Mixed Convection Heat Transfer From a Horizontal Plate With Variable Surface Heat Flux in a Porous Medium," Heat and Mass Transfer, Vol. 34, PP. 1-7, 1998.

5. Oosthuizen, P. H., "Mixed Convective Heat Transfer From a Heated Horizontal Plate in a Porous Medium Near an Impermeable Surface," Transactions of the ASME, Journal of Heat Transfer, Vol. 110, No. 2, PP. 390-394, May 1988.

6. Prasad, V., Lai, F.-C., and Kulacki, F.A., "Mixed Convection in Horizontal Porous Layers Heated From Below," Transactions of the ASME, Journal of Heat Transfer, Vol. 110, No. 2, PP. 395-402, May 1988.

7. Aldoss, T. K., Chen, T. S., and Armaly, B. F., "Mixed Convection Over Nonisothermal Horizontal Surfaces in a Porous Medium: the Entire Regime," Numerical Heat Transfer, Part A: Applications, Vol. 25, No. 6, PP. 685-701, 1994.

8. Chen, C.-H., "Analysis of Non-Darcian Mixed Convection From Impermeable Horizontal Surfaces in Porous Media: the Entire Regime," International Journal of Heat Mass Transfer, Vol. 40, No. 12, PP. 2993-2997, 1997.

9. Gorla, R. S. R., Shanmugam, K., and Kumari, M., "Nonsimilar Solutions for Mixed Convection in Non-Newtonian Fluids Along Horizontal Surfaces in Porous Media," Transport in Porous Media, Vol. 28, PP. 319-334, 1997.

10. Gorla, R. S. R., Shanmugam, K., and Kumari, M., "Mixed Convection in Non-Newtonian Fluids Along Nonisothermal Horizontal Surfaces in Porous Media," Heat and Mass Transfer, Vol. 33, PP. 281-286, 1998.

11. Thiele, M., "Heat Dispersion in Stationary Mixed Convection Flow About Horizontal Surfaces in Porous Media," Heat and Mass Transfer, Vol. 33, PP. 7-16, 1997.

12. Duwairi, H. M., Aldoss, T. K., and Jarrah, M. A., "Nonsimilarity Solutions for Non-Darcy Mixed Convection From Horizontal Surfaces in a Porous Medium," Heat and Mass Transfer, Vol. 33, PP. 149-156, 1997.

13. Kumari, M., and Nath, G., "Non-Darcy Mixed Convection in Power-Law Fluids Along a Non-isothermal Horizontal Surface in a Porous Medium," International Journal of Engineering Science, Vol. 42, PP. 353-369, 2004.

14. Hassanien, I. A., and Omer, Gh. M., "Mixed-Convection Flow Adjacent to a Horizontal Surface in a Porous Medium With Variable Permeability and Surface Heat Flux," Journal of Porous Media, Vol. 8, No. 2, PP. 225-235, 2005.

15. Khanafer, K., and Vafai, K., "Double-Diffusive Mixed Convection in a Lid-Driven Enclosure Filled With a Fluid-Saturated Porous Medium," Numerical Heat Transfer, Part A, Vol. 42, PP. 465-486, 2002.

16. Hassanien, I. A., and Omer, Gh. M., "Nonsimilarity Solutions for Mixed Convection Flow Along Nonisothermal Vertical Plate Embedded in Porous Media With Variable Permeability," Journal of Porous Media, Vol. 5, No. 2, PP. 159-167, 2002.

17. Mohammadein, A. A., and El-Shaer, N. A., "Influence of Variable Permeability on Combined Free and Forced Convection Flow Past a Semi-Infinite Vertical Plate in a Saturated Porous Medium," Heat and Mass Transfer, Vol. 40, PP. 341-346, 2004.

18. Ibrahim, F. S., and Hassanien, I. A., "Influence of Variable Permeability on Combined Convection Along a Nonisothermal Wedge in a Saturated Porous Medium," Transport in Porous Media, Vol. 39, PP. 57-71, 2000.

19. Mansour, M. A., and El-Shaer, N. A., "Mixed Convection-Radiation in Power-Law Fluids Along a Non-Isothermal Wedge in a Porous Medium With Variable Permeability," Transport in Porous Media, Vol. 57, PP. 333-346, 2004. 
20. Marcondes, F., Medeiros, J. M., and Gurgel, J. M., "Numerical Analysis of Natural Convection in Cavities With Variable Porosity," Numerical Heat Transfer, Part A, Vol. 40, PP. 403-420, 2001.

21. Nield, D. A., and Bejan, A., Convection in Porous Media, Third Edition, Springer Science + Business Media, Inc., 2006.

22. Mohammad, S. A., "Nonsimilar Coupled Heat and Mass Transfer in Mixed Convection Flow Along a Horizontal Permeable Plate Embedded in a Saturated Porous Medium With Variable Permeability," Ph.D. Thesis, College of Engineering, University of Mosul, 2008.

23. Mansour, M. A., and Gorla, R. S. R., "Mixed Convection-Radiation Interaction in PowerLaw Fluids Along a Nonisothermal Wedge Embedded in a Porous Medium," Transport in Porous Media, Vol. 30, PP. 113-124, 1998. 